\title{
A Comprehensive Performance Evaluation of MIPv6 and PMIPv6 Mobility Management Protocols in Wireless Mesh Network
}

Original Scientific Papers

\author{
Wei Siang Hoh \\ Universiti Malaysia Pahang (UMP), \\ Faculty of Computing, \\ College of Computing \& Applied Sciences \\ Pekan, Pahang, Malaysia \\ weisiang@ump.edu.my
}

\author{
Bi-Lynn Ong \\ Universiti Malaysia Perlis (UniMAP), \\ Faculty of Electronic Engineering Technology, \\ Ulu Pauh, Perlis, Malaysia \\ drlynn@unimap.edu.my
}

\author{
Si-Kee Yoon \\ Universiti Malaysia Perlis (UniMAP), \\ Faculty of Electronic Engineering Technology, \\ Ulu Pauh, Perlis, Malaysia \\ sikeeyoon@gmail.com
}

\author{
R Badlishah Ahmad \\ Universiti Malaysia Perlis (UniMAP), \\ Ulu Pauh, Perlis, Malaysia \\ badli@unimap.edu.my
}

\begin{abstract}
Wireless communication is becoming essential due to the dramatic increase in the usage of mobile devices. The high demand for real-time or instant services requires wireless Internet networks which can support different Quality of Service (QoS) guarantees and different traffic characteristics. All Internet network mobile device services are supported by mobility management protocols. In this paper, we compare the performance of the MIPv6 and PMIPv6 mobility management protocols in the Wireless Mesh Network (WMN) environment. We identify and analyze the MIPv6 and PMIPv6 mobility management protocols' characteristics by using performance indicators. The performance comparison of MIPv6 and PMIPv6 mobility management protocols was conducted in terms of throughput, latency, and packet loss ratio. Based on the conducted experimental results, we summarize the performances for MIPV6 and PMIPv6 mobility management protocols in the Wireless Mesh Network environment. The results obtained indicate that PMIPv6 generally outperforms MIPv6. In future work, the evaluation of HMPIv6, FMIPv6, and FHMIPv6 is proposed.
\end{abstract}

Keywords: PMIPv6, MIPv6, Network-Based \& Host-Based Mobility Management Protocol, Wireless Mesh Network

\section{INTRODUCTION}

In recent years, the number of Internet users in wireless environments has grown tremendously, causing network distortion issues. A pressing issue is that mobile wireless ecosystems have proliferated rapidly in the wireless environment [1]. These wireless ecosystems play an important role in mobility management protocols. This has led to various mobility management protocols for enabling mobility services. Mobility support protocols can be divided into two main categories: host-based and network-based. Mobile Internet Protocol version 6 (MIPv6) [2], and its enhancements, such as Fast Handover Mobile Internet Protocol version 6 (FMIPv6) [3], Hierarchical Mobile Internet Protocol version 6 (HMIPv6) [4] and Fast Handover for Hierarchical Mobile Internet Protocol version 6 (FHMIPv6), are categorized as host-based. Network-based mobility management protocols have been designed and introduced to address the shortcomings of host-based mobility management protocols [5].
Network-based protocols include Fast Proxy Mobile Internet Protocol version 6 (FPMIPv6) and Proxy Mobile Internet Protocol version 6 (PMIPv6) [6].

In MIPv6, the Mobile Node (MN) plays an important role in the mobile scenario. MN allows the alteration of its network attachment points without disturbing IP packet delivery to or from the node. Access Network Procedures are introduced to maintain the current location of all the MNs in the network. PMIPv6 allows an MN to alter its point of attachment without requiring mobility signaling to be processed at MN [5]. Hence, IP packet delivery is not interrupted, and the $\mathrm{MN}$ remains reachable in the topology. There are two types of mobility service provisioning entities: Local Mobility Anchor (LMA) and Mobility Access Gateway (MAG). Fast Proxy Mobile IPv6 (FPMIPv6) [6] was designed and introduced to increase the handover performance by preventing the loss of packets and reducing latency during the handover. Fig. 1 depicts the design structure of network-based and hostbased mobility systems. 


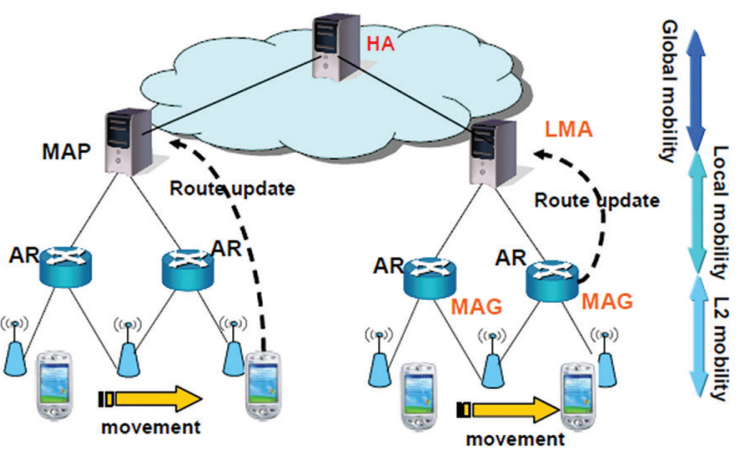

Host-based mobility

Fig. 1. Network-based vs. Host-based mobility

In this research paper, the characteristics and performance profiles of PMIPv6 and MIPv6 are analyzed in the WMN topology environment. These two mobility management protocols have been designed, developed, and analyzed. The performance parameters include Packet Delivery Ratio (PDR), throughput, and latency. The paper content is organized as follows: related works, terminology, simulation design, results, and finally the conclusion.

\section{RELATED WORKS}

M. Skořepa et al. [7] investigated "Analytical Method for L3 handover Latency Evaluation". The researchers conducted an analytical comparison based on MIPv6 handover schemes. They completed the comparison for the four most common handover schemes, including the cost of packet delivery of FHMIPv6, FMIPv6, HMIPv6, and MIPv6. The research access network focused on the IEEE $802.11 \mathrm{~b}$ family. The transport of the core network was focused on Ethernet (IEEE 802.3 100BaseT). The researchers implemented the analytical methods to obtain the comparison results. Handover cost and handover latency were taken into account by the researchers in the main performance matrices.

A. Ahmad and D. Sasidharan [8] investigated "Handover efficiency improvement in Proxy Mobile IPv6 (PMIPv6) networks". The researchers aimed to reduce the handover delay for PMIPv6 through a communication state-dependent chaining scheme. Chaining based PMIPv6 (CBPMIPv6) was introduced, in which the Mobility Access Gateways (MAGs) were chained to support movements within the PMIPv6 domain. The analytical simulation was conducted using NS2. The results demonstrated that using buffering schemes was able to reduce the packet loss and that handover latency can be reduced through a triggering scheme.

Yan Zhang, et al. [9] investigated "The Simulation of Hierarchical Mobile Ipv6 with Fast Handover using NS2". The researchers performed simulation in the Network Simulator version 2 (NS2) software. Four types of mobile routing protocols were compared to determine which offered the best performance for the mobile network. These included FHMIPv6, HMIPv6, FMIPv6,

and MIPv6. Ultimately, FHMIPv6 performed the best against other MIPs in terms of jitter and handover delay. However, this research did not present or discuss any potential reason for the low performances of the other MIPs.

W.K. Jia [10] investigated "A unified MIPv6 and PMIPv6 route optimization scheme for heterogeneous mobility management domains". The researchers designed a unified approach to Route Optimization (RO) scheme. This scheme is based on a simplified MIPv6 Return Routability Procedure (RRP) protocol. It is called Traffic Driven Pseudo Binding Update (TDPBU). The analytical framework of TDPBU for performance analysis included signaling cost, end-to-end latency, throughput, and other performance metrics. The proposed scheme ensured immediate route optimization without considering the residing location of MNs in the heterogeneous MIPv6/PMIPv6 environment. In conclusion, the TDPBU was able to significantly enhance the overall performance of mobility management schemes.

S. Muthut et al. [11] investigated the performance of MIPv6, HMIPv6, and FMIPv6 with WMN. The performance matrix included end-to-end delay, throughput, and packet delivery ratio. The MIPv6, FMIPv6, and HMIPv6 all perform inter-handover to measure the performances under the same network condition. The result showed that HMIPv6 performed better than FMIPv6 and MIPv6 in terms of throughput and packet delivery ratio. In terms of end-to-end delay, MIPv6 outperformed FMIPv6 and HMIPv6 when the network only performed inter-handover. The low end-to-end delay attained by MIPv6 with WMN contributed to low throughput and PDR. However, the authors focused only on performance evaluation. There were no proposed improvements or enhancement schemes to overcome the problems found in their research.

A. Yadav and A. Singh [12] performed performance analysis and optimization of FMIPv6 and HMIPv6 handover. The researchers proposed a new analytical model for the MIPv6 optimization protocol. The researchers used MATLAB 7 software to complete the simulation by collecting a sample size of 100, for both FMIPv6 and HMIPv6. According to the researchers, the new analytical model could reduce the handover by $50 \%$ of the original MIP working mechanism. In conclusion, the proposed analytical method is able to significantly reduce handover latency in HMIPv6 and FMIPv6. However, the researchers were only concerned with handover latency as the sole performance metric. The results obtained from this research may have been affected by the mobile node moving with random motion.

\section{TERMINOLOGY}

In this section, all the general terms are discussed in detail. MIPv6 and PMIPv6 protocols, WMN characteristics and behaviors, and handover management are also discussed in detail. 


\subsection{MOBILITY MANAGEMENT CLASSIFICATION}

Mobility management enables a mobile device on a data network to change the attachment point. At the same time, the mobile device will have an IP address, which is also known as Mobile Host (MH). A change in IP address can be a big challenge to maintain uninterrupted data flow, ensure security, minimize loss of packets, and identify the newer location. Mobility management can be classified into four types, which are cross layer, upper layer, network layer, and lastly link layer mobility management [13]. In the network layer mobility management, it is further subclassified into 2 main groups, which are micro \& macro mobility and host-based \& network-based mobility. Fig. 2 below represents the classification of mobility management [14].

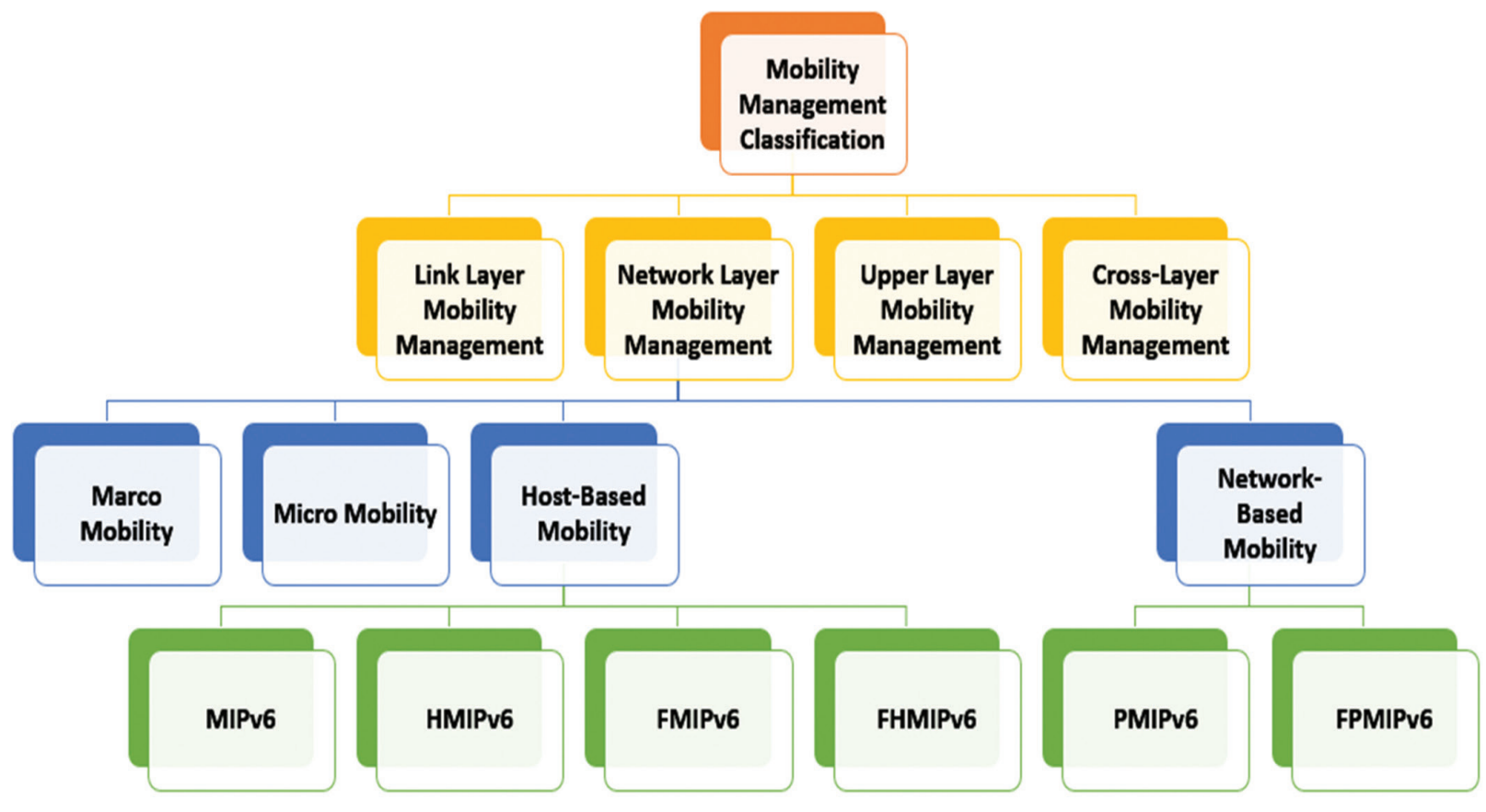

Fig. 2. Mobility Management [14]

\subsection{MOBILE INTERNET PROTOCOL VERSION-6 (MIPV6)}

The Internet Engineering Task Force (IEFT) established the use of Mobile Internet Protocol version 6 (MIPv6). The mechanism of MIPv6 allows MN to be reachable and to maintain an ongoing connection, even though its position keeps on changing within the topology. During the entire process, $\mathrm{MN}$ is still able to maintain the same allocated IP address [15]. Once the operation is started, the MN will be searching for Foreign Agent (FA) and detecting its movement. It will autoconfigure and set up itself with a New Care of Address (NCoA) through either a stateless or stateful mechanism. Binding Update (BU) is sent by $M N$ and it will forward to its Home Agent (HA) to notify its new address which is available. The $\mathrm{HA}$ returns Binding Acknowledgment (BAck). With the help of HA, all packets are tunneled to MN's NCoA. Route Optimization (RO) is another mode for MIPv6. The RO will search for the shortest path and start to transfer the packets. This process requires $\mathrm{MN}$ to register its current Binding to Corresponding Node (CN). The $\mathrm{CN}$ allows the delivery of the triangulate packets to $\mathrm{MN}$ without getting permission from HA. In conclusion, it is able to decrease the congestion at MN's HA and Home Link [16]. Fig. 3 below represents the messages flow of MIPv6.

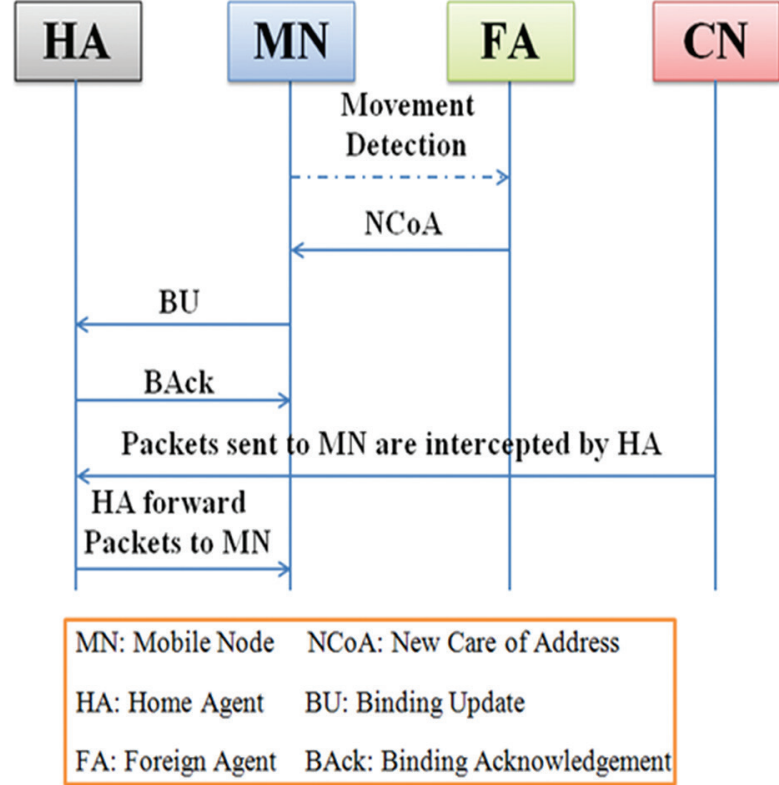

Fig. 3. MIPv6 Flow Diagram

\subsection{PROXY MOBILE INTERNET PROTOCOL}

\section{VERSION-6 (PMIPV6)}

PMIPv6 was developed based on MIPv6's [RFC3775] design. It avoids tunneling over head over the air, 
which may cause latency to increase dramatically. Such latency can be seen in MIPv6 [17]. The operation begins when Mobile Node (MN) moves and attaches to an access router which is called Mobile Access Gateway (MAG). Once the authentication is completed, MAG identifies the MN. The MAG obtains the MN's profile, which contains the Home Address and sends the Proxy Binding Update (PBU) to the Localized Mobility Agent
(LMA) on behalf of MN. If MAG receives the Proxy Binding Acknowledgment ( $A C K$ ) from LMA, then it sends Router Advertisements that contain MN's home network prefix. If MAG does not receive Proxy Binding Acknowledgment (ACK) from LMA, it waits and resends the Proxy binding Update to LMA. Fig. 4 below provides an overview of PMIPv6.

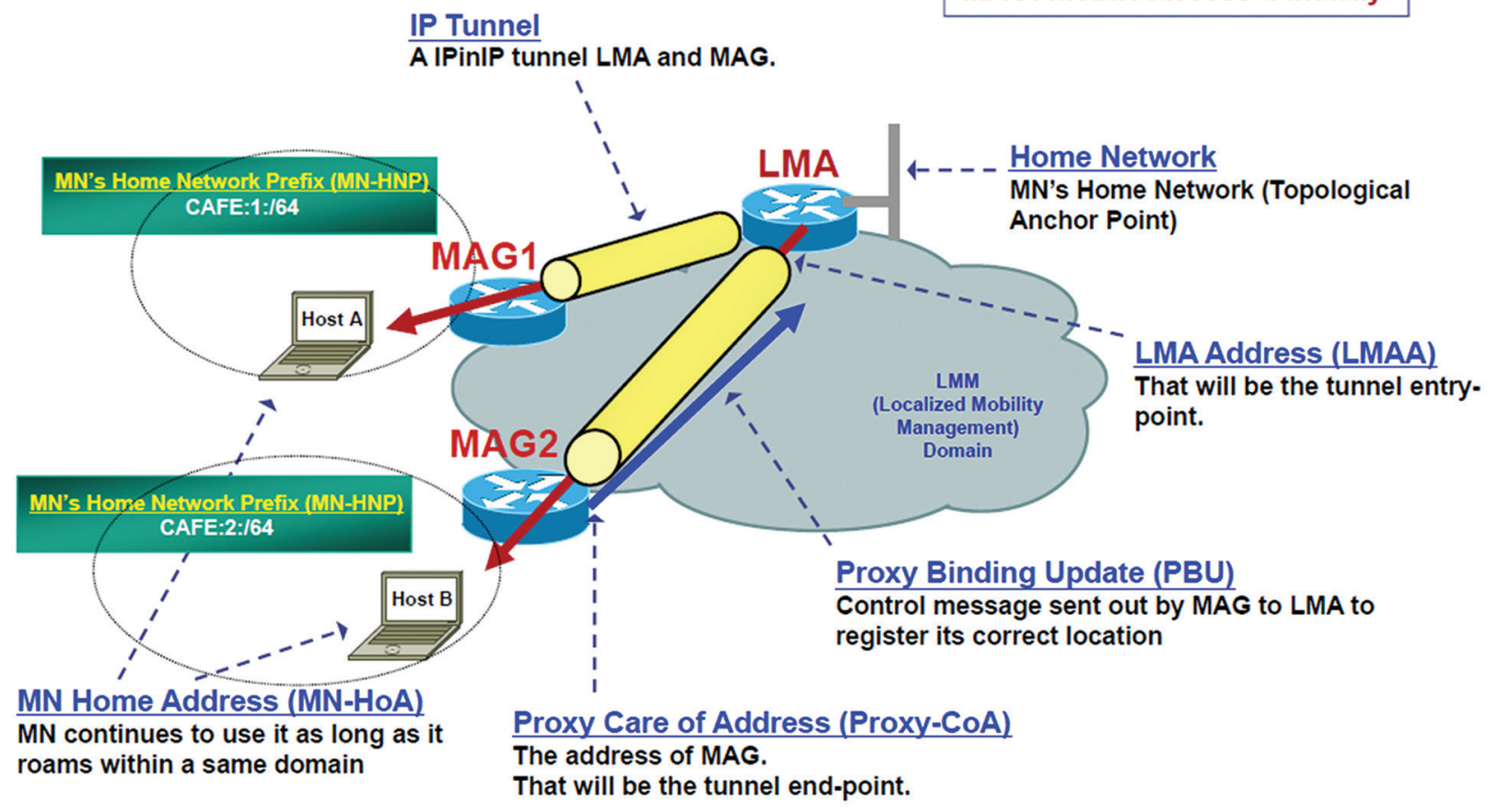

LMA: Localized Mobility Agent MAG: Mobile Access Gateway

\section{Fig. 4. PMIPv6 Protocols [18}

This concludes the discussion of the classification of mobility management, mobility management protocols, MIPv6, and PMIPv6. Table 1 shows the summarized characteristics of MIPv6 and PMIPv6. In this paper, MIPv6, and PMIPv6 are analysed in terms of packet delivery ratio, latency, and throughput.

Table 1. The summarized characteristic of MIPV6 and PMIPv6 in each protocols criteria

\begin{tabular}{|ccc}
\hline Protocol Criteria & MIPv6 & PMIPv6 \\
\hline Mobility Scope & Global Mobility & Local Mobility \\
\hline Location Management & Yes & No \\
\hline Required Infrastructure & Home Agent (HA) & LMA, MAG \\
\hline MN Modification & Yes & No \\
\hline Handover Latency & Poor & Good \\
\hline Localized Routing & Yes & No \\
\hline Tunneling Over Wireless Link & Required & Not Required \\
\hline Route Advertisement Type & Broadcast & Unicast \\
\hline Return Routability & Required & Not Required
\end{tabular}

Wireless Mesh Network (WMN) is defined as an infrastructure-based network. A WMN is a communications network which has various wireless nodes which are sorted in a mesh topology. WMN consists of gateways, mesh routers, and mesh clients. Mobile phones, laptops, tablets, intelligent machines, and other wireless devices are the examples of mesh clients. Mesh routers forward traffic to and from the gateway, but are not connected to the Internet.

The total coverage area of the radio nodes functioning as a single network is defined as a mesh cloud. The mesh cloud is dependent on the radio nodes, which operate in harmony with each other to create a radio network. WMN offers trust and provides good redundancy. When one single node cannot operate or is damaged, the rest of the nodes are still connected and the communication is maintained[19].

Fig. 5 shows how a WMN can self-organize and selfconfigure without any command from the network operator. WMN can be implemented with various wireless technologies, such as IEEE 802.11s WiFi Mesh and IEEE 802.16 WiMAX. A telecommunication company can expand, replace, and adapt their network based on the requests of the end users [20]. 


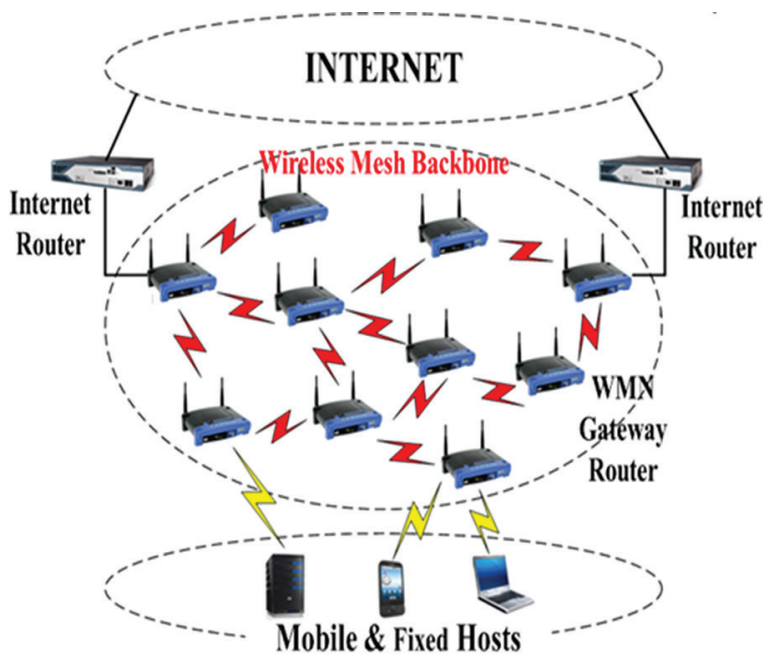

Fig. 5. Wireless Mesh Network Architecture [20]

A WMN can provide a more planned and systematic configuration. The deployment of WMN can provide cost-effective and dynamic connectivity over forested or mountainous areas. The network architecture consists of a mesh router that is able to support large areas at a low cost compared to single-hop routers. An alternate routing or dynamic connectivity allows traffic loads to be balanced and minimizes bottlenecks. This may also be able to significantly increase network reliability in WMN. Table 2 shows the difference between WMN and Wireless Ad Hoc Network (WANET).

Table 2. Difference between WMN and WANET

\begin{tabular}{|ccc|}
\hline Issues & WANET & WMN \\
\hline $\begin{array}{c}\text { Infrastructure } \\
\text { requirement }\end{array}$ & Infrastructure-less & $\begin{array}{c}\text { Partial or Fully Fixed } \\
\text { Infrastructure }\end{array}$ \\
\hline Network Topology & Highly Dynamic & Relatively Static \\
\hline Energy Constraint & High & Low \\
\hline $\begin{array}{c}\text { Application } \\
\text { Characteristic }\end{array}$ & Temporary & $\begin{array}{c}\text { Semi-permanent or } \\
\text { Permanent }\end{array}$ \\
\hline Routing Performance & Fully Distributed & $\begin{array}{c}\text { Fully or Partially } \\
\text { Distributed }\end{array}$ \\
\hline Geographical & Do Not Consider & Well Perform \\
\hline
\end{tabular}

\subsection{MOBILITY MANAGEMENT IN WMN}

As mentioned above, WMN serves as an access network that implements multi-hop wireless forwarding. Hence, the nonmobile nodes relay data to and from the Internet. IETF has also announced that WMN can provide a data transmission rate up to $134.4 \mathrm{Mbps}$. Hence, it is capable of satisfying the requirements of next generation wireless networks with high speed and low latency. The commercialization of WMN is inevitable. Several working groups focus on the WMN technologies and corresponding specifications (e.g., IEEE 802.16a and 802.11) are being standardized. However, mobility management for mobile users in WMN is not specified clearly.

\subsection{HANDOVER MANAGEMENT}

A handoff or handover is one of the essential parts of mobility management. These terms refer to the same process of changing the point of the connection while a call is in progress. The objective of a handover is to provide seamless handover between mobile terminal and BS. A smooth handover can minimize the loss of data, while a fast handover can decrease the delay to and provide seamless handover service. A handover is needed to meet user preferences. Handover can be classified into two main categories, namely horizontal handover (intracell) and vertical handover (intercell). The main differences between horizontal handover and vertical handover are complexity and symmetry. Due to the different access technologies and their diverse characteristics, a vertical handover is asymmetric and more complex than a horizontal handover.

\section{SIMULATION DESIGN}

In this research paper, the MIPv6 and PMIPv6 protocols were designed, developed, and simulated in Network Simulator version 2 (NS2). Both mobility management protocols are set up in the same WMN environment for comparison and analysis.

\subsection{THE PARAMETER OF THE NETWORK TOPOLOGY DESIGN}

In order to compare the two different types of mobility management protocols, a few configurations and parameters need to be in constant value to obtain the optimum results for both mobility management protocols. The network environment for both mobility management protocols is set up to Mac 802.11. The data rate is fixed to $10 \mathrm{Mb}$. The interface queue type is drop tail mode.

After setting up the wireless environment, the number of nodes of MIPv6 and PMIPv6 needs to be built up. For MIPv6, the number of nodes is 5 nodes and these consist of one Home Agent (HA) and one Client Node (CN). For PMIPv6, it consists of one Home Agent (HA), one Client Node (CN), one LMA and two MAGs. Table 3 represents the detail of the parameter and its values for setting up the MIPv6 and PMIPv6 mobility management protocols.

Table 3. Type of parameters and value

\begin{tabular}{cc}
\hline Link Delay & $2 \mathrm{~ms}$ \\
\hline Data Rate for Mac 802.11 & $10 \mathrm{Mb}$ \\
\hline Window Size (Byte) & 32 \\
\hline Duration & $100 \mathrm{~s}$ \\
\hline Transport Protocol & $\mathrm{TCP}$
\end{tabular}




\subsection{PERFORMANCE METRICS}

The characteristics and behavior of the network topology of PMIPv6 and MIPv6 can be understood through few performance metrics. The metrics are:

1. Latency mean, which represents the delay when the packet sent from the source passes through the router and base station to the destination; and

2. Throughput, which represents the total data transmitted from one source to receiver in time duration and is normally measured in kilobits per second (Kbps).
3. Packet Delivery Ratio (PDR) represents the ratio of received packets with sent packets between the receiver and source.

\section{RESULTS \& DISCUSSION}

Simulation results are presented with detailed discussion. Based on Table 4, each performance metrics result for MIPv6 and PMIPv6 are presented in detail. The packet size starts from 256 bytes, and increases to 512 bytes, 1024 bytes, 2048 bytes, and ends at 4096 bytes.

Table 4. Results of MIPv6 and PMIPv6 for different performance metrics

\begin{tabular}{|c|c|c|c|c|c|}
\hline \multicolumn{6}{|c|}{ Mobile Internet Protocols version 6 (MIPv6) } \\
\hline Packet Size (Bytes) & 256 & 512 & 1024 & 2048 & 4096 \\
\hline Latency Mean (ms) & 90 & 92 & 93 & 103 & 104 \\
\hline Throughput (Kbps) & 1.080 & 2.212 & 4.444 & 10.609 & 21.381 \\
\hline Packet Delivery Ratio (PDR) & 75.90 & 76.87 & 76.68 & 82.48 & 82.86 \\
\hline \multicolumn{6}{|c|}{ Proxy Mobile Internet Protocols version 6 (PMIPv6) } \\
\hline Packet Size (Bytes) & 256 & 512 & 1024 & 2048 & 4096 \\
\hline Latency Mean (ms) & 2 & 2 & 2 & 2 & 3 \\
\hline Throughput (Kbps) & 129.823 & 234.322 & 390.124 & 592.323 & 799.130 \\
\hline Packet Delivery Ratio (PDR) & 99.83 & 99.82 & 99.86 & 99.75 & 99.75 \\
\hline
\end{tabular}

Based on the observation of Table 4, when packet size increases from 256 bytes to 4096 bytes, the throughput increases gradually. The throughput for 256 bytes packet size is $1.080 \mathrm{kbps}$ and reaches $21.381 \mathrm{kbps}$ when the packet size increases to 4096 bytes. For PMIPv6, the throughput for packet size 256 bytes is $129.823 \mathrm{kbps}$, increasing gradually to $799.13 \mathrm{kbps}$ when the packet size reaches 4096 bytes.

Based on Fig. 6, the throughput between MIPv6 and PMIPv6 has a huge difference. The reason is in the basic MIPv6 protocol. In this protocol, during switching between different subnets, the MN needs to go through mobile testing, setting, getting the new address configuration, duplicating address detection, and finally binding the registration process. These processes cause a lot of switching delay, high rate of packet loss, and overload signaling. Throughput is very low due to these reasons. For PMIPv6, since it is completely transparent to mobile nodes, the MAG becomes the proxy and communicates with the Mobile Node. This can lower the probability of signaling overload and decrease the packet loss rate. The throughput of PMIPv6 is very high and it is completely utilized the bandwidth.

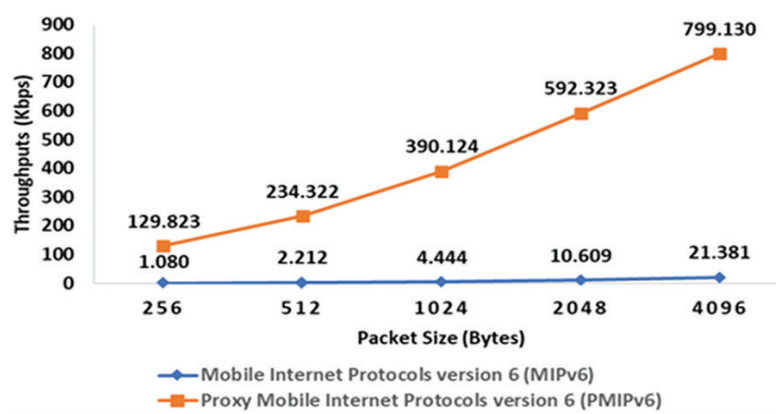

Fig. 6. A comparison of MIPv6 and PMIPv6 in terms of throughput (kbps)

Fig. 7 showed the packet delivery ratio of MIPv6 and PMIPv6 for various packet sizes. For MIPv6, the packet delivery ratio increases from $75.90 \%$ to $76.87 \%$ when the packet size is increased from 256 bytes to 512 bytes. When the packet size reaches 1024 bytes, the packet delivery ratio is slightly decreased to $76.68 \%$. This may be caused by the signaling overload. When packet size increases to 2048 bytes and 4096 bytes, the packet delivery ratio is $82.48 \%$ and $82.86 \%$ respectively. As compared to PMIPv6, the packet delivery ratio for ev- 
ery packet size is higher than MIPv6. When packet size is 256 bytes, the packet delivery ratio is $99.85 \%$ and it decreases to $99.82 \%$ when the packet size is 512 bytes. The packet delivery ratio slightly increases to $99.86 \%$ when the packet size is 1024 bytes. When packet size increases to 2048 bytes and 4096 bytes, the packet delivery ratio is constant at $99.75 \%$. In conclusion, PMIPv6 performed better than MIPv6 in terms of packet delivery ratio.

This is the reason why MIPv6 is used in global networks while PMIPv6 is used in localized networks. In global networks, the handoff procedure is not efficient and causes large latency. The packet drops when it reaches its timeout in TCP transmission. In localized networks where PMIPv6 is implemented, the limited topology contributes to minimal handoff signaling delays, low latency, and lower probability for packet drop due to the timeout. Hence, this explains the packet delivery ratio of MIPv6 is lower compared with PMIPv6.

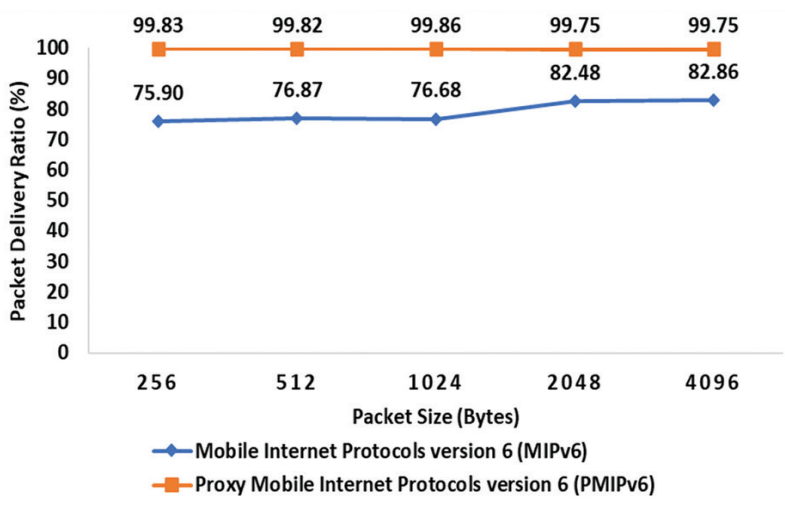

Fig. 7. A comparison of MIPv6 and PMIPv6 in terms of aspect packet delivery ratio (\%)

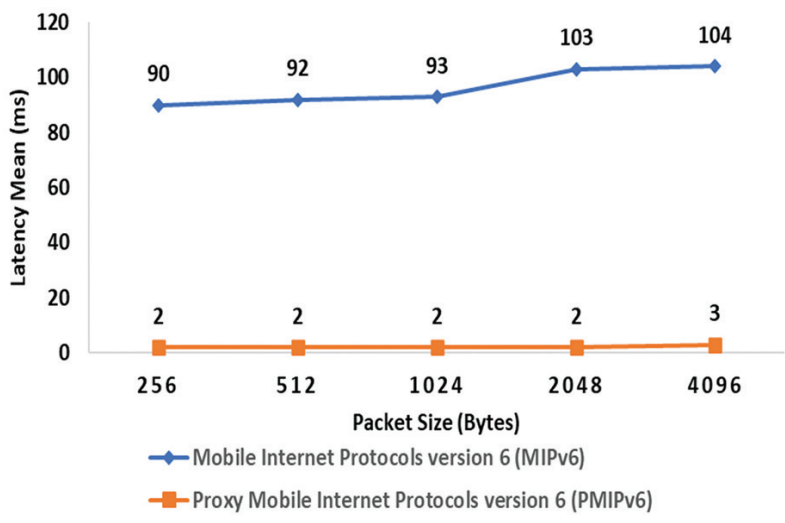

Fig. 8. A comparison of MIPv6 and PMIPv6 in terms of aspect latency mean (ms)

Figure 8 shows that the latency of the MIPv6 is very high compared with PMIPv6. For MIPv6, the latency reaches $90 \mathrm{~ms}$ when the packet size is 256 bytes; this increases to $92 \mathrm{~ms}, 93 \mathrm{~ms}, 103 \mathrm{~ms}$, and a peak of $104 \mathrm{~ms}$ when the packet size increases from 512 bytes, 1024 bytes, 2048 bytes, and 4096 bytes respectively. As for PMIPv6, the latency stays constant at $2 \mathrm{~ms}$ when the packet size increases from 256 bytes, 512 bytes, 1024 bytes, and 2048 bytes. The highest latency for PMIPv6 is $3 \mathrm{~ms}$ when the packet size reaches 4096 bytes.

The high latency of MIPv6 is caused by the handoff procedure. The handoff latency of MIPv6 mainly comes from the process of localized routing, modification of Mobile Node (MN), and return routing. These steps or processes in MIPv6 are excluded from PMIPv6. Thus, the latency of PMIPv6 is lower as compared to MIPv6.

\section{CONCLUSION}

In this research, a comparison between MIPv6 and PMIPv6 was made, and PMIPv6 has been shown to outperform MIPv6. The evaluation shows that PMIPv6 offers better throughput, lower latency, and higher PDR. The basic MIPv6 has various problems which are not able to fulfill the huge demand of mobile users. Hence, PMIPv6 is developed and implemented to overcome the drawback of MIPv6. For future works, the researcher suggests various modified handover methods to overcome this bottleneck. In future, the HMIPv6, FMIPv6, and FHMIPv6 with route optimization schemes should be compared with each other. The proposed methods are believed to outperform MIPv6, and are able to be used in preparation for the wireless network to $5 \mathrm{G}$ network technologies.

\section{REFERENCES:}

[1] A. B. Waluyo, W. Rahayu, D. Taniar, B. Scrinivasan, "A Novel Structure and Access Mechanism for Mobile Data Broadcast in Digital Ecosystems", IEEE Transactions on Industrial Electronics, Vol. 58, No. 6, 2011, pp. 2173-2182.

[2] C. Perkins, J. Arkko, D. Johnson, "Mobility Support in IPv6", IETF RFC 3775, 2004.

[3] R. Koodli, "Fast Handovers for Mobile IPv6", IETF RFC 4068, 2005.

[4] H. Soliman, C. Castelluccia, K. Elmalki, L. Bellier, "Hierarchical mobile IPv6 mobility management (HMIPv6)", RFC 4140, p. 5380, 2008.

[5] K. Chowdhury, K. Leung, B. Patil, V. Devarapalli, S. Gundavelli, “Proxy Mobile IPv6”, RFC 5213, 2008.

[6] H. Yokota, K. Chowdhury, R. Koodli, B. Patil, F. Xia, "Fast handovers for proxy mobile IPv6", RFC 5949, 2010.

[7] M. Skořepa, R. Klügl, "Analytical method for L3 handover latency evaluation", Proceedings of the European conference of systems, and European conference of circuits technology and devices, 
and European conference of communications, and European conference on Computer science, Tenerife, Spain, 30 November 2010, pp. 342-347.

[8] A. Ahmad, D. Sasidharan, "Handover efficiency improvement in Proxy Mobile IPv6 (PMIPv6) networks", Procedia Computer Science, Vol. 46, 2015, pp. 1064-1071.

[9] Y. Zhang, H. Bi, "The simulation of Hierarchical Mobile IPv6 with fast handover using NS2", Procedia Engineering, Vol. 37, 2012, pp. 214-217.

[10] W. K. Jia, "A unified MIPv6 and PMIPv6 route optimization scheme for heterogeneous mobility management domains", Computer Networks., Vol. 75, 2014, pp. 160-176.

[11] S. Muthut, B.-L. Ong, N. A. H. Zahri, R. B. Ahmad, "An overview of performance enhancement of FHMIPv6 on wireless mesh network", International Journal of Future Computer and Communication, Vol. 4, No. 3, 2015, pp. 160-164.

[12] A. Yadav, A. Singh, "Performance analysis and optimization Of Hmipv6 and Fmipv6 handoff management protocol", International Journal of Engineering Research, Vol. 5, No. 3, 2014, pp. 305-308.

[13] W. S. Hoh, S. Muthut, B.-L. Ong, M. Elshaikh, M. N. M. Warip, R. B. Ahmad, "A survey of mobility management protocols", ARPN Journal of Engineering and Applied Sciences, Vol 10, No. 19, 2015, pp. 9015-9019.

[14] W. S. Hoh, B.-L. Ong, R. B. Ahmad, H. Ahmad, "Consolidation of Host Based Mobility Management Protocols with Wireless Mesh Network", Innovative Computing, Optimization and Its Applications, pp. 111-129, Springer, 2018.
[15] X. Wu, G. Nie, "Comparison of different mobility management schemes for reducing handover latency in Mobile IPv6", Proceedings of the International Conference on Industrial Mechatronics and Automation, Chengdu, China, 15-16 May 2009, pp. 256-259.

[16] S. Shayma, I. Mahamod, J. Kasmiran, "A comparison of mobile node 's handoff between mobile IPv6 and fast handover protocol", The Institution of Engineers Malaysia, 2008.

[17] S. Y. Ren, R. Chai, L. Tang, Q. B. Chen, "Proxy Mobile IPv6 based inter-domain mobility management approach and performance analysis", Application Research of Computers, Vol 27, No. 3, 2010, pp.1118-1121.

[18] N. Neumann, J. Lei, X. Fu, G. Zhang, "I-PMIP: an inter-domain mobility extension for proxy-mobile IP", Proceedings of the International Conference on Wireless Communications and Mobile Computing: Connecting the World Wirelessly, Leipzig, Germany, 21-24 June 2009, pp. 994-999.

[19] Z. L. Lee, B.-L. Ong, A. Amir, W. S. Hoh, "A survey of session initiation protocol in Wireless Mesh Network", Proceedings of the IEEE $15^{\text {th }}$ Student Conference on Research and Development, Wilayah Persekutuan Putrajaya, Malaysia, 13-14 December 2017, pp. 286-290.

[20] S. Muthut, B.-L. Ong, W. S. Hoh, R. B. Ahmad, "Integration of fast handover and hierarchical mobile internet protocol with wireless mesh network", Australian Journal of Basic and Applied Sciences, Vol 9, No. 25, 2015, pp. 72-78. 